\title{
A Retrospective Study of Cytology, High-Risk HPV, and Colposcopy Results of Vaginal Intraepithelial Neoplasia Patients
}

\author{
Qing Cong, ${ }^{1,2,3}$ Yu Song, ${ }^{1,2,3}$ Qing Wang, ${ }^{1,2,3}$ Hongwei Zhang $\mathbb{D}$, , \\ Shujun Gao, ${ }^{1,2,3}$ and Long Sui $\mathbb{D}^{1,2,3}$ \\ ${ }^{1}$ Obstetrics and Gynecology Hospital of Fudan University, Shanghai, China \\ ${ }^{2}$ Shanghai Key Laboratory of Female Reproductive Endocrine Related Diseases, Shanghai, China \\ ${ }^{3}$ Shanghai Medical Center of Key Programs for Female Reproductive Diseases, Shanghai, China \\ Correspondence should be addressed to Long Sui; suilonggyn@163.com
}

Received 5 January 2018; Revised 15 March 2018; Accepted 2 April 2018; Published 8 May 2018

Academic Editor: Marcelo A. Soares

Copyright (C) 2018 Qing Cong et al. This is an open access article distributed under the Creative Commons Attribution License, which permits unrestricted use, distribution, and reproduction in any medium, provided the original work is properly cited.

\begin{abstract}
There is currently no large sample data of cytology, high-risk human papillomavirus (hrHPV), and colposcopy results of vaginal intraepithelial neoplasia (VaIN) in women who underwent hysterectomy and those who did not. We aim to explore the values of cytology, hrHPV, and colposcopy reports in detecting VaIN. A retrospective study of women diagnosed with VaIN by colposcopydirected biopsy was performed at the Obstetrics and Gynecology Hospital of Fudan University, China, between January 1, 2014, and December 31, 2014. A total of 529 cases of VaIN were diagnosed, including 16.1\% VaIN2/3 and 83.9\% VaIN1. The ratio of VaIN2/3 in VaIN among patients after hysterectomy and with an intact uterus was $35.1 \%$ and $12.0 \%$, respectively. The sensitivity of cytology for VaIN2/3 in only, concomitant, and posthysterectomy VaIN was $42.1 \%, 80.0 \%$, and $80.8 \%$, respectively. The sensitivity of hrHPV and cytology/hrHPV cotesting for VaIN2/3 in patients with an intact uterus versus those after hysterectomy was $93.5 \%$ versus $92.3 \%$ and $92.0 \%$ versus $100.0 \%$, respectively. Notably, $13.3 \%$ of the patients with VaIN and $9.7 \%$ of the patients with VaIN2/3 underwent hysterectomy for noncervical diseases. The sensitivity of cytology and hrHPV for VaIN is noninferior to that of CIN2+, and thus these methods can help in the early detection of VaIN effectively.
\end{abstract}

\section{Introduction}

Vaginal cancer is a human papillomavirus- (HPV-) associated gynecologic disease, accounting for approximately $1 \%$ to $4 \%$ of cancers of the female genital tract. High-grade squamous intraepithelial lesion (HSIL) or vaginal intraepithelial neoplasia (VaIN) grade $2 / 3$ is a precancerous lesion analogous to HSIL/cervical intraepithelial neoplasia (CIN) grade 2/3 [1]. Low-grade squamous intraepithelial lesion (LSIL) or VaIN 1 is a benign manifestation of HPV infection. The natural history of VaIN is thought to be similar to that of CIN. In the past, VaIN was rarer than vaginal invasive cancer because it was frequently underdiagnosed $[2,3]$. The reported incidence rate of vaginal cancer is 0.4 to 0.6 per 100,000 women, while the incidence of VaIN is $0.2-0.3$ per 100,000 women [4-6]. The reported frequencies were $0.5 \%$ of all neoplastic lower genital tract lesions [7].
Over recent decades, the diagnosis of vaginal intraepithelial neoplasia (VaIN) has increased steadily as a result of widespread application of cytology/high-risk human papilloma virus (hrHPV) cotesting and colposcopy in cervical cancer screening. In the colposcopy clinic of the largest obstetrics and gynecology tertiary teaching hospital in China, the detection rate of VaIN in all lower genital tract intraepithelial lesions was $11 \%(1,923 / 16,732)$ on average, with an increasing trend from 2013 to 2015 [8]. However, few studies have investigated the cytology, hrHPV, and colposcopy results in VaIN. The number of VaIN cases included in currently available studies was limited to 6 to 132 cases, and most focused on posthysterectomy patients [5, 9-14], because current cervical cancer screening guidelines recommend that women who have had cervical precancer or invasive cervical cancer undergo continued surveillance testing for at least 20 years after treatment [15]. Besides, many women after total 
hysterectomy for benign diseases undergo vaginal cytology and/or hrHPV tests, and clinicians are faced with dilemmas of managing their abnormal results [16]. Should women after total hysterectomy for benign diseases be referred to colposcopy or just leave it? The meaning or value of these abnormal results needs to be investigated.

Up till now, there is limited data on cytology, hrHPV, and colposcopy of VaIN in women who underwent hysterectomy and those who did not. In our hospital, the largest obstetrics and gynecology tertiary teaching hospital in China, women, including those who underwent hysterectomy and those who did not, undergo regular cytology and/or hrHPV testing; those with abnormal screening reports are referred to colposcopy. On this basis, a large retrospective study of VaIN patients was performed to explore the values of cytology, hrHPV, and colposcopy in detecting VaIN, which might help understand clinical characterization of VaIN, including distribution of VaIN1 and VaIN2/3, cytology/hrHPV sensitivity, and indications of previous hysterectomy of VaIN. To the best of our knowledge, this is the largest retrospective study of cytology and hrHPV results in VaIN to date.

\section{Methods}

All women diagnosed with VaIN by colposcopy-directed biopsy between January 1, 2014, and December 31, 2014, at the Obstetrics and Gynecology Hospital of Fudan University were included. VaIN was histologically diagnosed by two independent gynecologic pathologists. Women with abnormal cytology but normal histological diagnosis were excluded. Approval was obtained from the institutional review board of the Obstetrics and Gynecology Hospital of Fudan University before data extraction was performed and consent to research signed. All available data including demographics, history, histological information, cytology, and hrHPV testing results were recorded. Bethesda System terminology was used for reporting cytology results [17]. In 529 patients with VaIN, 517 had complete medical history data. Their original composition of vaginal, cervical, and vulvar lesions was listed when history of cervical or vulvar lesions was considered (Table 1).

There were 222 patients with only VaIN, because 41 of 263 patients with only VaIN included in Table 3 had a history of cervical or vulvar conization or laser ablation. In total, $42.9 \%$ of the patients had only VaIN and $57.1 \%$ had concomitant cervical or vulvar lesions. And $15.9 \%$ of the cases were VaIN2/3 and $84.1 \%$ were VaIN1.

Regular cytology and/or hrHPV screening are performed in all women after hysterectomy. Women with abnormal cytology or hrHPV reports were referred to colposcopy in our hospital. Women with a prior hysterectomy performed for cervical lesions were routinely referred to colposcopy at least once. Complete history was available in 83 patients with VaIN (VaIN2/3, 37.3\%; VaIN1, 62.7\%) after hysterectomy. Table 2 shows the indications for previous hysterectomy in patients with VaIN. Among these, $86.7 \%$ underwent hysterectomy for cervical lesions, including cervical cancer (30.1\%) and precancer (56.6\%); 13.3\% underwent hysterectomy for
TABLE 1: Original composition of vaginal, cervical, and vulvar lesions when history was considered.

\begin{tabular}{lccc}
\hline Vagina & Cervix & Vulva & Number \\
\hline HSIL & $/$ & $/$ & 18 \\
HSIL & CA & $/$ & 14 \\
HSIL & HSIL & $/$ & 35 \\
HSIL & LSIL & $/$ & 12 \\
HSIL & LSIL & HSIL & 1 \\
HSIL & $/$ & HSIL & 2 \\
LSIL & $/$ & $/$ & 204 \\
LSIL & CA & $/$ & 14 \\
LSIL & HSIL & $/$ & 56 \\
LSIL & LSIL & $/$ & 154 \\
LSIL & LSIL & CA & 1 \\
LSIL & LSIL & HSIL & 5 \\
LSIL & $/$ & HSIL & 1 \\
Total & & & 517 \\
\hline
\end{tabular}

HSIL: high-grade squamous intraepithelial lesion; LSIL: low-grade squamous intraepithelial lesion; / stands for no lesion.

noncervical lesions, including uterine fibroid, endometrial cancer, ovarian cancer, and fallopian tube cancer.

We used Thinprep 2000 (TCT) or AutoCyte/PrepStain (LCT) for cytology testing, and Hybrid Capture 2 assay (Qiagen, Hilden, Germany) or Cobas 4800 assay (Roche, Penzberg, Germany) for HR-hrHPV testing. Chi-square tests were performed with SPSS 16.0 software (IBM, New York, USA). A $P$ value $<0.05$ was considered to be statistically significant.

\section{Results}

3.1. Classification of 529 Cases of VaIN. Based on a history of hysterectomy and the presence of only VaIN or VaIN concomitant with cervical or vulvar lesions, 529 cases of VaIN were classified as reported in Table 3. The mean age of the 529 patients with VaIN was $46.0 \pm 12.6$ years (range: $20-79$ ); $16.1 \%$ were diagnosed with VaIN2/3 with a mean age of $50.4 \pm 12.2$ years (range: $20-72$ ) and $83.9 \%$ were diagnosed with VaIN1 with a mean age of $45.1 \pm 12.6$ years (range: $21-79$ ).

Among 435 patients without a history of hysterectomy, $12.0 \%$ were diagnosed with VaIN $2 / 3$ and $88.0 \%$ were diagnosed with VaIN1; $60.5 \%$ were diagnosed with only VaIN and $39.5 \%$ were diagnosed with VaIN concomitant with cervical or vulvar lesions. Among patients with only VaIN, 9.1\% were VaIN2/3 and $90.9 \%$ were VaIN1. Among those with concomitant VaIN, $16.3 \%$ were VaIN $2 / 3$ and $83.7 \%$ were VaIN1. In 94 patients with a history of hysterectomy, 35.1\% were diagnosed with VaIN2/3 and $64.9 \%$ with VaIN1. The ratio of VaIN2/3 among patients with concomitant cervical lesions was higher than that of patients with only VaIN $(P=$ $0.04)$; the ratio of VaIN2/3 among patients after hysterectomy was higher than that of patients without hysterectomy $(P=$ $0.00)$ 
TABLE 2: Indications and rates of previous hysterectomy in 83 VaIN patients.

\begin{tabular}{|c|c|c|c|c|c|c|}
\hline \multirow{2}{*}{ Indications of hysterectomy } & \multicolumn{2}{|c|}{ VaIN } & \multicolumn{2}{|c|}{ VaIN2/3 } & \multicolumn{2}{|c|}{ VaIN1 } \\
\hline & Number & $\%$ & Number & $\%$ & Number & $\%$ \\
\hline Cervical lesions & 72 & $86.7 \%$ & 28 & $90.3 \%$ & 44 & $84.6 \%$ \\
\hline Cervical cancer & 25 & $30.1 \%$ & 11 & $35.5 \%$ & 14 & $26.9 \%$ \\
\hline Cervical precancer & 47 & $56.6 \%$ & 17 & $54.8 \%$ & 30 & $57.7 \%$ \\
\hline Noncervical lesions & 11 & $13.3 \%$ & 3 & $9.7 \%$ & 8 & $15.4 \%$ \\
\hline Endometrial cancer & 2 & $2.4 \%$ & 2 & $6.5 \%$ & 0 & $0.0 \%$ \\
\hline Fallopian tube cancer & 1 & $1.2 \%$ & 0 & $0.0 \%$ & 1 & $1.9 \%$ \\
\hline Ovarian cancer & 1 & $1.2 \%$ & 0 & $0.0 \%$ & 1 & $1.9 \%$ \\
\hline Myoma & 7 & $8.4 \%$ & 1 & $3.2 \%$ & 6 & $11.5 \%$ \\
\hline Total & 83 & $100.0 \%$ & 31 & $100.0 \%$ & 52 & $100.0 \%$ \\
\hline
\end{tabular}

VaIN: vaginal intraepithelial neoplasia.

TABLE 3: VaIN diagnosed in patients after hysterectomy and with no hysterectomy.

\begin{tabular}{lcc}
\hline VaIN & Number & Rate \\
\hline No hysterectomy & 435 & $100.0 \%$ \\
VaIN2/3 & $52 / 435$ & $12.0 \%$ \\
VaIN1 & $383 / 435$ & $88.0 \%$ \\
\hline Only vaginal lesions & $263 / 435$ & $60.5 \%$ \\
VaIN2/3 & $24 / 263$ & $9.1 \%$ \\
VaIN1 & $239 / 263$ & $90.9 \%$ \\
\hline Concomitant lesions & $172 / 435$ & $39.5 \%$ \\
VaIN2/3 & $28 / 172$ & $16.3 \%$ \\
VaIN1 & $144 / 172$ & $83.7 \%$ \\
\hline After hysterectomy & 94 & $100.0 \%$ \\
VaIN2/3 & $33 / 94$ & $35.1 \%$ \\
VaIN1 & $61 / 94$ & $64.9 \%$ \\
\hline
\end{tabular}

VaIN: vaginal intraepithelial neoplasia.

3.2. Cytology Results of 405 Cases of VaIN. According to the history of hysterectomy and the diagnosis of only or concomitant VaIN, available cytology reports of 405 cases of VaIN were classified as shown in Table 4. Cytology reports of VaIN can include negative diagnoses for intraepithelial lesion or malignancy (NILM), atypical squamous cells of undetermined significance (ASC-US), LSIL, atypical squamous cells that cannot exclude HSIL (ASC-H), or HSIL.

In 326 cases of VaIN without hysterectomy, the sensitivity of cytology was $59.5 \%$; among these, $10.4 \%$ were VaIN2/3 and sensitivity of cytology for VaIN2/3 was $58.8 \%$. In 212 cases of only VaIN, sensitivity of cytology was $52.4 \%$. Among these, $8.96 \%$ were VaIN2/3 and sensitivity of cytology was $42.1 \%$. In 114 cases of concomitant VaIN, sensitivity of cytology was $72.8 \%$. Among these, $13.2 \%$ were VaIN2/3 and sensitivity of cytology was $80.0 \%$. In 79 cases of VaIN after hysterectomy, sensitivity of cytology was $69.6 \%$. Among these, $32.9 \%$ were VaIN2/3 and sensitivity of cytology was $80.8 \%$.

3.3. Sensitivity of Cytology, hrHPV, and Cytology/hrHPV Cotesting in 529 Patients with VaIN. A total of 405 cytology reports were available; sensitivity of cytology for the diagnosis of VaIN is reported in Table 5. In 425 patients with VaIN and no history of hysterectomy, 326 cytology reports were available and the sensitivity of cytology for VaIN, VaIN1, and VaIN2/3 was 59.5\%,59.6\%, and 58.8\%, respectively. In 94 patients with VaIN after hysterectomy, 79 cytology reports were available and the sensitivity of cytology for VaIN, VaIN1, and VaIN2/3 was $69.6 \%, 64.2 \%$, and $80.8 \%$, respectively.

A total of $349 \mathrm{hrHPV}$ reports were available (Table 5). In 425 patients with VaIN and no history of hysterectomy, 276 hrHPV reports were available and the sensitivity of hrHPV for VaIN, VaIN1, and VaIN2/3 was $86.5 \%, 85.7 \%$, and $93.5 \%$, respectively. In 94 patients with VaIN after hysterectomy, 73 hrHPV reports were available and sensitivity of hrHPV for VaIN, VaIN1, and VaIN2/3 was 90.4\%, 89.4\%, and 92.3\%, respectively.

In 529 patients with VaIN, 326 cytology/hrHPV cotesting reports were available (Table 5). In 425 patients with VaIN and no history of hysterectomy, 260 cotesting reports were available and sensitivity of cotesting for VaIN, VaIN1, and VaIN2/3 was $91.9 \%, 91.9 \%$, and $92.0 \%$, respectively. In 94 patients with VaIN after hysterectomy, 66 cotesting reports were available and sensitivity of cotesting for VaIN, VaIN1, and VaIN2/3 was $98.5 \%, 97.7 \%$, and $100.0 \%$, respectively.

\section{Discussion}

Ratio of VaIN2/3 is higher in concomitant VaIN than in only VaIN and higher in patients after hysterectomy than in patients without hysterectomy (Table 3). In our study, 529 patients with VaIN were included and $17.8 \%$ had a history of hysterectomy, including $83.9 \%$ VaIN1 and 16.1\% VaIN2/3. Zhang et al. retrospectively analyzed 152 patients with VaIN, and $39.5 \%$ of the patients had a history of hysterectomy [18], including $45.4 \% \mathrm{VaIN} 1$ and $54.6 \% \mathrm{VaIN} 2 / 3$. The reason why the ratio of VaIN2/3 in their study is higher than ours might be because the ratio of posthysterectomy patients in their study was higher than that in ours $(P=0.00)$.

Cytology sensitivity was higher in VaIN after hysterectomy than in only VaIN without hysterectomy and higher in concomitant VaIN than in only VaIN without hysterectomy (Table 4). The rates of both VaIN and VaIN2/3 after hysterectomy were higher than in only VaIN and VaIN2/3 without 
TABLE 4: Detailed cytology results of VaIN diagnosed in patients after hysterectomy and with no hysterectomy.

\begin{tabular}{|c|c|c|c|c|c|c|c|}
\hline \multirow{2}{*}{ VaIN } & \multicolumn{6}{|c|}{ Cytology number (\%) } & \multirow{2}{*}{ Sensitivity (\%) } \\
\hline & NILM & ASC-US & LSIL & ASC-H & HSIL & $\mathrm{CA}$ & \\
\hline VaIN with uterus & $132(40.5 \%)$ & $71(21.8 \%)$ & $108(33.1 \%)$ & $6(1.8 \%)$ & $8(2.5 \%)$ & $1(0.3 \%)$ & $59.5 \%$ \\
\hline VaIN2/3 & $14(41.2 \%)$ & $7(20.6 \%)$ & $10(29.4 \%)$ & $1(2.9 \%)$ & $1(2.9 \%)$ & $1(2.9 \%)$ & $58.8 \%$ \\
\hline VaIN1 & $118(40.4 \%)$ & $64(21.9 \%)$ & $98(33.6 \%)$ & $5(1.7 \%)$ & $7(2.4 \%)$ & $0(0.0 \%)$ & $59.6 \%$ \\
\hline Only VaIN & $101(47.6 \%)$ & $50(23.6 \%)$ & $56(26.4 \%)$ & $3(1.4 \%)$ & $2(0.9 \%)$ & $0(0.0 \%)$ & $52.4 \%$ \\
\hline VaIN2/3 & $11(57.9 \%)$ & $4(21.1 \%)$ & $3(15.8 \%)$ & $1(5.3 \%)$ & $0(0.0 \%)$ & $0(0.0 \%)$ & $42.1 \%$ \\
\hline VaIN1 & $90(46.6 \%)$ & $46(23.8 \%)$ & $53(27.5 \%)$ & $2(1.0 \%)$ & $2(1.0 \%)$ & $0(0.0 \%)$ & $53.4 \%$ \\
\hline Concomitant VaIN & $31(27.2 \%)$ & $21(18.4 \%)$ & $52(45.6 \%)$ & $3(2.6 \%)$ & $6(5.3 \%)$ & $1(0.9 \%)$ & $72.8 \%$ \\
\hline VaIN2/3 & $3(20.0 \%)$ & $3(20.0 \%)$ & $7(46.7 \%)$ & $0(0.0 \%)$ & $1(6.7 \%)$ & $1(6.7 \%)$ & $80.0 \%$ \\
\hline VaIN1 & $28(28.3 \%)$ & $18(18.2 \%)$ & $45(45.5 \%)$ & $3(3.0 \%)$ & $5(5.1 \%)$ & $0(0.0 \%)$ & $71.7 \%$ \\
\hline VaIN after hysterectomy & $24(30.4 \%)$ & $16(20.3 \%)$ & $24(30.4 \%)$ & $4(5.1 \%)$ & $11(13.9 \%)$ & $0(0.0 \%)$ & $69.6 \%$ \\
\hline VaIN2/3 & $5(19.2 \%)$ & $3(11.5 \%)$ & $3(11.5 \%)$ & $4(15.4 \%)$ & $11(42.3 \%)$ & $0(0.0 \%)$ & $80.8 \%$ \\
\hline VaIN1 & $19(35.8 \%)$ & $13(24.5 \%)$ & $21(39.6 \%)$ & $0(0.0 \%)$ & $0(0.0 \%)$ & $0(0.0 \%)$ & $64.2 \%$ \\
\hline
\end{tabular}

VaIN: vaginal intraepithelial neoplasia; NILM: negative for intraepithelial lesion or malignancy; ASCUS: atypical squamous cells of undetermined significance; LSIL: low-grade squamous intraepithelial lesion; ASC-H: atypical squamous cells cannot exclude HSIL; HSIL: high-grade squamous intraepithelial lesion; CA: cancer.

TABLE 5: Cytology/hrHPV sensitivity in VaIN patients after hysterectomy and with no hysterectomy.

\begin{tabular}{lccc}
\hline VaIN & No hysterectomy & After hysterectomy & $P$ value \\
\hline Cytology sensitivity & & & \\
VaIN & $59.5 \%(194 / 326)$ & $69.6 \%(55 / 79)$ & 0.098 \\
VaIN1 & $59.6 \%(174 / 292)$ & $64.2 \%(34 / 53)$ & 0.532 \\
VaIN2/3 & $58.8 \%(20 / 34)$ & $80.8 \%(21 / 26)$ & \\
hrHPV sensitivity & & & \\
VaIN & $86.5 \%(239 / 276)$ & $90.4 \%(66 / 73)$ & 0.070 \\
VaIN1 & $85.7 \%(210 / 245)$ & $89.4 \%(42 / 47)$ & 0.382 \\
VaIN2/3 & $93.5 \%(29 / 31)$ & $92.3 \%(24 / 26)$ & \\
Cotesting sensitivity & & & 0.855 \\
VaIN & $91.9 \%(239 / 260)$ & $98.5 \%(65 / 66)$ & 0.058 \\
VaIN1 & $91.9 \%(216 / 235)$ & $97.7 \%(43 / 44)$ & 0.170 \\
VaIN2/3 & $92.0 \%(23 / 25)$ & $100.0 \%(22 / 22)$ & 0.175 \\
\hline
\end{tabular}

VaIN: vaginal intraepithelial neoplasia; hrHPV: high-risk human papillomavirus.

hysterectomy $(P=0.01)$, respectively, which demonstrates that the sensitivity of cytology for VaIN after hysterectomy was higher than the sensitivity of cytology for only VaIN and similar to that for concomitant VaIN without hysterectomy. In patients without hysterectomy, the sensitivity of cytology was higher for concomitant VaIN, VaIN2/3, and VaIN1 than for only VaIN, VaIN2/3, and VaIN1 $(P=0.00, P=0.03$, and $P$ $=0.00)$, respectively. In our view, cytology sampling of VaIN concomitant with cervical lesions includes abnormal cervical cells in addition to abnormal vaginal cells, and cervical cells make up the largest component of exfoliative cells. Thus, their sensitivity of cytology is higher for concomitant VaIN than isolated VaIN. For women after hysterectomy, all cytology samples come from the vagina, and thus the sensitivity of cytology for VaIN was higher than that of isolated VaIN in patients without hysterectomy.

Cytology and hrHPV sensitivity for VaIN were noninferior to sensitivity for CIN2+ (CIN2 or worse) and might be higher in women after hysterectomy. Cytology and hrHPV tests are used to screen cervical lesions. Our study showed that both of these methods can be used to screen for VaIN, especially among women after hysterectomy, because they showed noninferior sensitivity in comparison to women without hysterectomy. In the ATHENA trial, the sensitivity of cytology for CIN2+ was $40.6 \%$ (95\% confidence interval [CI]: $36.1-45.1 \%)$ in women $\geq 25$ years of age [19]; liquid-based cytology specimens from 46,887 eligible women $\geq 21$ years of age were evaluated at four large regional US laboratories, and there were considerable differences among the laboratories both in the overall rates of cytological abnormalities, ranging from 3.8 to $9.9 \%$, and in the sensitivity of cytology to detect CIN2+, from 42.0 to $73.0 \%$. In contrast, the hrHPV positivity rate varied only from 10.9 to $13.4 \%$, and the sensitivity of hrHPV testing varied from 88.2 to $90.1 \%$ [20]. In another study, cytology sensitivity for CIN2+ was 39.5\% (95\% CI: 29.4-49.5\%), Hybrid Capture 2 (HC2) hrHPV sensitivity for CIN2+ was 93.2\% (95\% CI: 87.1-99.2\%), and Aptima hrHPV sensitivity for CIN2+ was $87.8 \%$ (95\% CI: 80.2-95.5\%) [21]. 
In our study, cytology sensitivity for VaIN2/3 was $58.8-80.8 \%$ and hrHPV sensitivity for VaIN2/3 was $92.3-93.5 \%$, whether there was a history of hysterectomy or not, which showed that the sensitivity of cytology and hrHPV for VaIN was noninferior to the sensitivity for CIN2+, and these methods might be used to detect VaIN2/3 and recurrence of cervical cancer in the vaginal apex.

Vaginal or vulvar lesions could be more severe than cervical lesions. In concomitant VaIN, cervical lesions (two locations of the lower genital tract) represented $96.6 \%$, vulvar lesions (two locations) $1.0 \%$, and cervical and vulvar lesions (all three locations) $2.4 \%$ of VaIN (Table 1). Generally, the cervix is regarded as the most susceptible and severest location for intraepithelial neoplasia of the lower genital tract, which results in potential neglect in the evaluation of the vagina and vulva during colposcopy. In fact, the most severe lesions can be located in the vagina and vulva rather than the cervix. Therefore, careful examination of the entire lower genital tract in colposcopy is essential for the diagnosis of vaginal and vulvar lesions.

Approximately one-tenth of VaIN2/3 occurred after hysterectomy for noncervical diseases. Our data show that $13.3 \%$ of patients with VaIN and $9.7 \%$ of patients with VaIN2/3 had a history of hysterectomy for noncervical diseases. We believe that the actual ratio might be higher because many women stop cytology or hrHPV testing, according to the current guideline. Since there might be insufficient assessment of cervical lesions before hysterectomy and patients might be infected with hrHPV before or after hysterectomy, regular cytology or hrHPV testing can help detect VaIN2/3 and vaginal cancer in posthysterectomy patients. The available literature was too limited to develop evidence based recommendations for managing abnormal vaginal cytology and hrHPV screening tests. An algorithm based on expert opinion is proposed for managing women with abnormal vaginal test results [17].

\section{Conclusion}

The sensitivity of cytology and hrHPV for VaIN is noninferior to that of CIN2+, and thus these methods can help in the early detection of VaIN effectively.

\section{Conflicts of Interest}

The authors declare that they have no conflicts of interest.

\section{Authors' Contributions}

Qing Cong and Yu Song contributed equally to this study.

\section{Acknowledgments}

Data collection and interpretation were supported by the National Natural Science Foundation of China (81471423), Innovation Project of the Science and Technology Commission of Shanghai Municipality (16411950200), and Health Commission Project of Shanghai Municipality (20144Y0098,
201344095). The authors would like to thank Editage (https://www.editage.com) for English language editing. They also sincerely thank Evans Heather, Shakir Fevzi, Carmel Flynn, and Preeti Chadha from the Colposcopy Department of the Royal Free Hospital in the United Kingdom of Great Britain and Northern Ireland for constructive advice and manuscript modification.

\section{References}

[1] T. M. Darragh, T. J. Colgan, J. T. Cox et al., "The lower anogenital squamous terminology standardization project for hpv-associated lesions: Background and consensus recommendations from the college of american pathologists and the american society for colposcopy and cervical pathology," Journal of Lower Genital Tract Disease, vol. 16, no. 3, pp. 205242, 2012.

[2] A. Frega, F. Sopracordevole, C. Assorgi et al., "Vaginal intraepithelial neoplasia: A therapeutical dilemma," Anticancer Reseach, vol. 33, no. 1, pp. 29-38, 2013.

[3] M. Aho, E. Vesterinen, B. Meyer, E. Purola, and J. Paavonen, "Natural history of vaginal intraepithelial neoplasia," Cancer, vol. 68, no. 1, pp. 195-197, 1991.

[4] E. Diakomanolis, K. Stefanidis, A. Rodolakis et al., "Vaginal intraepithelial neoplasia: Report of 102 cases," European Journal of Gynaecological Oncology, vol. 23, no. 5, pp. 457-459, 2002.

[5] R. M. Rome and P. G. England, "Management of vaginal intraepithelial neoplasia: A series of 132 cases with long-term follow-up," International Journal of Gynecological Cancer, vol. 10, no. 5, pp. 382-390, 2000.

[6] F. H. Sillman, R. G. Fruchter, Y.-S. Chen, L. Camilien, A. Sedlis, and E. McTigue, "Vaginal intraepithelial neoplasia: Risk factors for persistence, recurrence, and invasion and its management," American Journal of Obstetrics \& Gynecology, vol. 176, no. 1, pp. 93-99, 1997.

[7] R. J. Cardosi, J. J. Bomalaski, and M. S. Hoffman, "Diagnosis and management of vulvar and vaginal intraepithelial neoplasia," Obstetrics and Gynecology Clinics of North America, vol. 28, no. 4, pp. 685-702, 2001.

[8] Q. Cong, Q. Wang, S. J. Gao et al., "Detection trend of vaginal intraepithelial neoplasia diagnosed by colposcopy guided biopsy from 2013 to 2015," Zhonghua Fu Chan Ke Za Zhi, vol. 52, pp. 239-243, 2017.

[9] K. F. Pearce, H. K. Haefner, S. F. Sarwar, and T. E. Nolan, "Cytopathological findings on vaginal Papanicolaou smears after hysterectomy for benign gynecologic disease," The New England Journal of Medicine, vol. 335, no. 21, pp. 1559-1562, 1996.

[10] A. Frega, D. French, J. Piazze, A. Cerekja, G. Vetrano, and M. Moscarini, "Prediction of persistent vaginal intraepithelial neoplasia in previously hysterectomized women by high-risk HPV DNA detection," Cancer Letters, vol. 249, no. 2, pp. 235241, 2007.

[11] K. A. So, J. H. Hong, J. H. Hwang et al., “The utility of the human papillomavirus DNA load for the diagnosis and prediction of persistent vaginal intraepithelial neoplasia," Journal of Gynecologic Oncology, vol. 20, pp. 232-237, 2009.

[12] M. Bansal, R. M. Austin, and C. Zhao, "Correlation of histopathologic follow-up findings with vaginal human papillomavirus and low-grade squamous intraepithelial lesion papanicolaou test results," Archives of Pathology \& Laboratory Medicine, vol. 135, no. 12, pp. 1545-1549, 2011. 
[13] F. Sopracordevole, F. Mancioli, N. Clemente et al., "Abnormal Pap Smear and Diagnosis of High-Grade Vaginal Intraepithelial Neoplasia: A Retrospective Cohort Study," Medicine, vol. 94, p. e1827, 2015.

[14] Z. Li, S. Barron, W. Hong, A. Karunamurthy, and C. Zhao, "Surveillance for recurrent cancers and vaginal epithelial lesions in patients with invasive cervical cancer after hysterectomy: Are vaginal cytology and high-risk human papillomavirus testing useful?" American Journal of Clinical Pathology, vol. 140, no. 5, pp. 708-714, 2013.

[15] D. Saslow, D. Solomon, H. W. Lawson et al., "American Cancer Society, American Society for Colposcopy and Cervical Pathology, and American Society for Clinical Pathology screening guidelines for the prevention and early detection of cervical cancer," CA: A Cancer Journal for Clinicians, vol. 62, no. 3, pp. $147-172,2012$.

[16] M. J. Khan, L. S. Massad, W. Kinney et al., "A common clinical dilemma: Management of abnormal vaginal cytology and human papillomavirus test results," Journal of Lower Genital Tract Disease, vol. 20, no. 2, pp. 119-125, 2016.

[17] D. Solomon, D. Davey, R. Kurman et al., "The 2001 Bethesda system: terminology for reporting results of cervical cytology," The Journal of the American Medical Association, vol. 287, no. 16, pp. 2114-2119, 2002.

[18] J. Zhang, X. Chang, Y. Qi, Y. Zhang, and S. Zhang, "A retrospective study of 152 women with vaginal intraepithelial neoplasia," International Journal of Gynecology and Obstetrics, vol. 133, no. 1, pp. 80-83, 2016.

[19] T. C. Wright, M. H. Stoler, C. M. Behrens, A. Sharma, G. Zhang, and T. L. Wright, "Primary cervical cancer screening with human papillomavirus: End of study results from the ATHENA study using HPV as the first-line screening test," Gynecologic Oncology, vol. 136, no. 2, pp. 189-197, 2015.

[20] T. C. Wright, M. H. Stoler, C. M. Behrens, A. Sharma, K. Sharma, and R. Apple, "Interlaboratory variation in the performance of liquid-based cytology: Insights from the ATHENA trial," International Journal of Cancer, vol. 134, no. 8, pp. 1835-1843, 2014.

[21] T. Iftner, S. Becker, K.-J. Neis et al., "Head-to-head comparison of the RNA-based aptima human papillomavirus (HPV) assay and the DNA-based hybrid capture $2 \mathrm{HPV}$ test in a routine screening population of women aged 30 to 60 years in Germany," Journal of Clinical Microbiology, vol. 53, no. 8, pp. 25092516, 2015. 


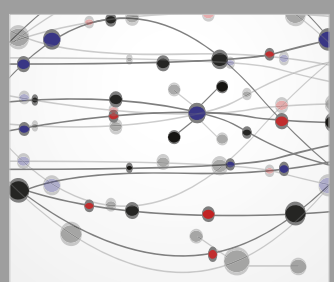

The Scientific World Journal
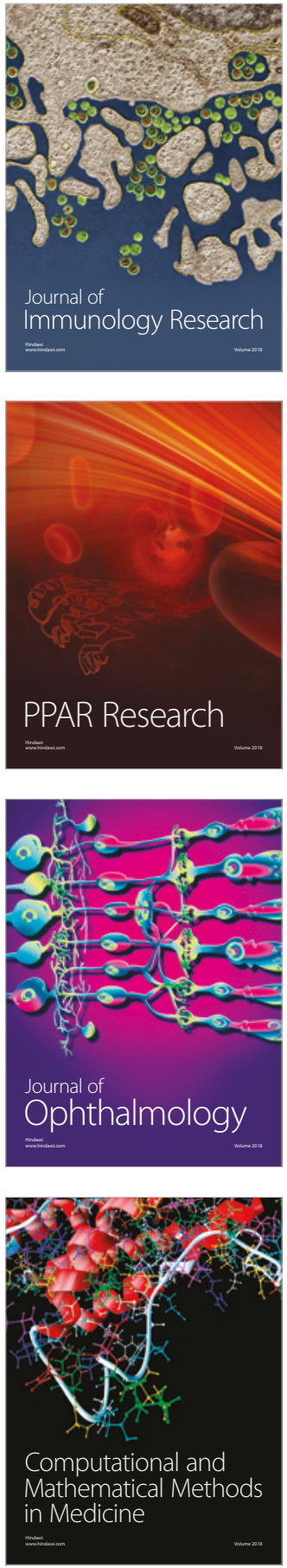

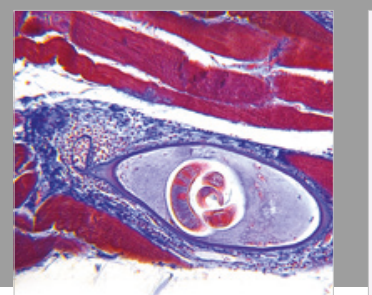

Gastroenterology Research and Practice

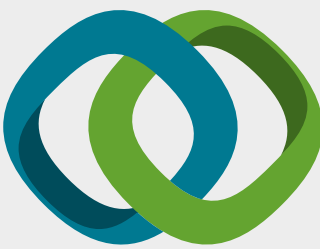

\section{Hindawi}

Submit your manuscripts at

www.hindawi.com
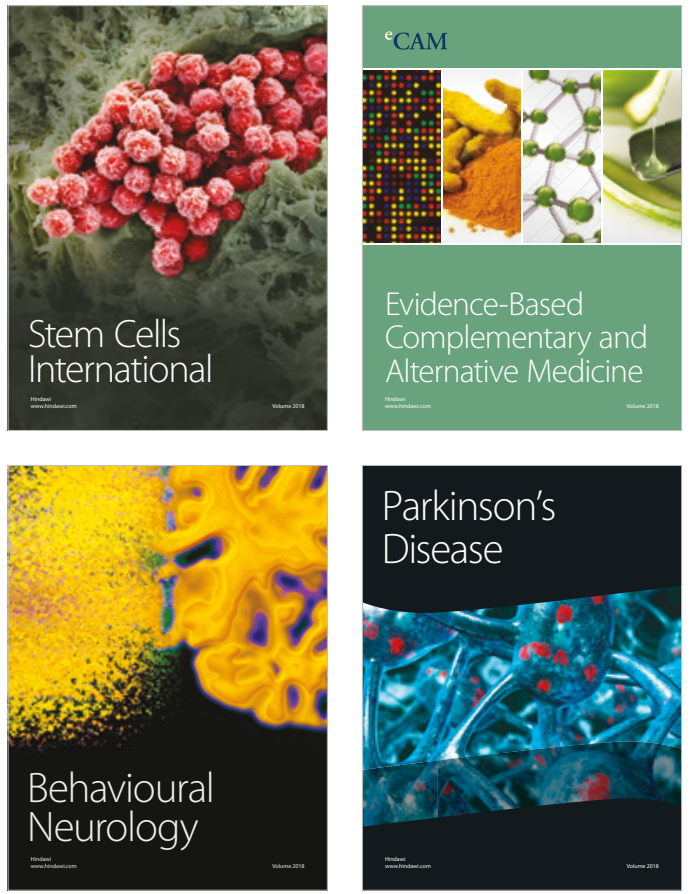

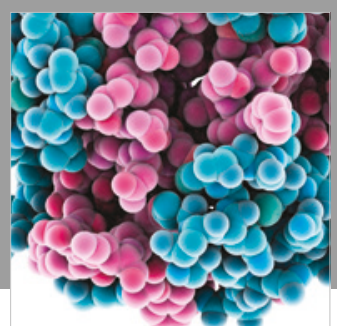

ournal of

Diabetes Research

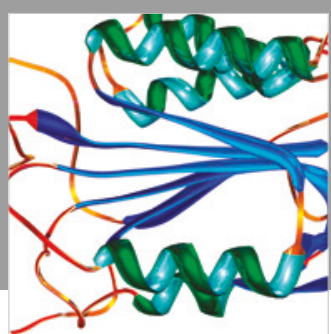

Disease Markers
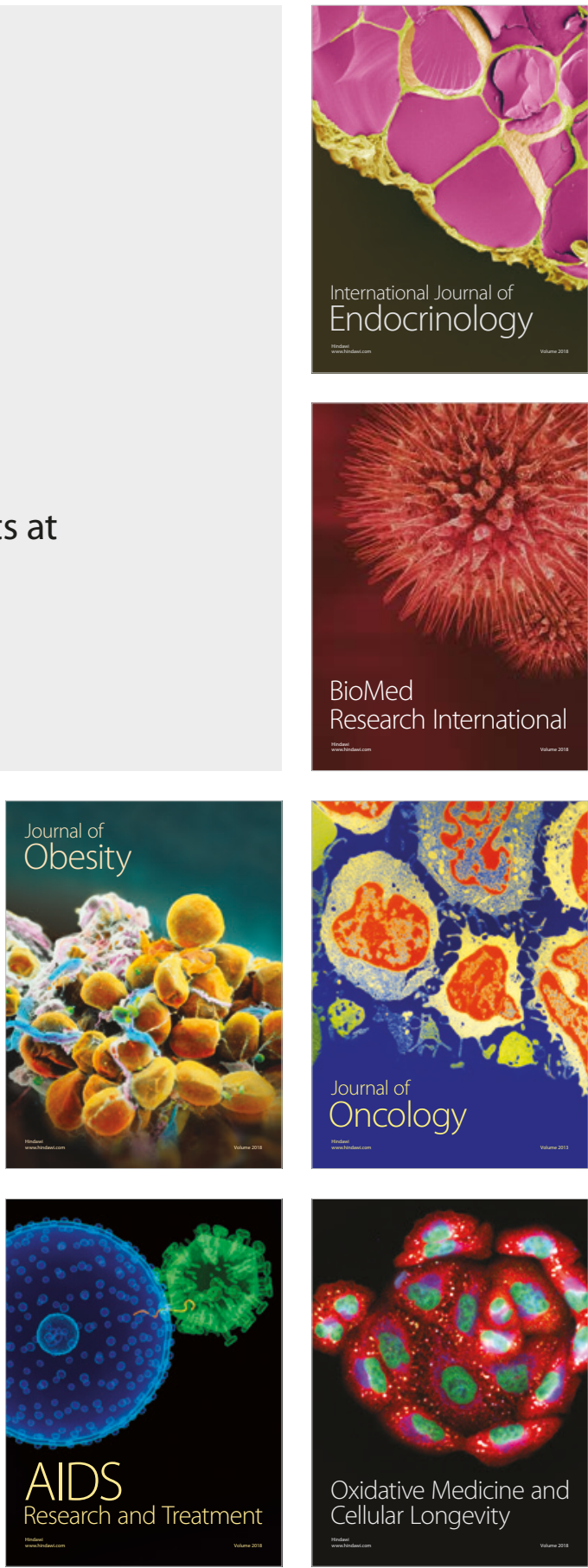